Editorial

\title{
State-of-the-Art Infrared Applications in Drugs, Dietary Supplements, and Nutraceuticals
}

\author{
Alessandra Durazzo $\mathbb{D D}^{1}$ Massimo Lucarini ${ }^{1}{ }^{1},{ }^{1}$ Johannes Kiefer ${ }^{(D)},{ }^{2}$ \\ and Sarfaraz Ahmed Mahesar $\mathbb{D i D}^{3}$ \\ ${ }^{1}$ CREA-Research Centre for Food and Nutrition, Via Ardeatina 546, Rome 00178, Italy \\ ${ }^{2}$ Technische Thermodynamik, Universität Bremen, Badgasteiner Str. 1, Bremen 28359, Germany \\ ${ }^{3}$ National Centre of Excellence in Analytical Chemistry, University of Sindh, Jamshoro-76080, Pakistan \\ Correspondence should be addressed to Alessandra Durazzo; alessandra.durazzo@crea.gov.it
}

Received 7 July 2019; Accepted 9 July 2019; Published 3 February 2020

Copyright (c) 2020 Alessandra Durazzo et al. This is an open access article distributed under the Creative Commons Attribution License, which permits unrestricted use, distribution, and reproduction in any medium, provided the original work is properly cited.

Infrared spectroscopy, including near-infrared (NIR) and mid-infrared (MIR), is an example of a green, fast, and environment-friendly technique. It is considered as an innovative and rapid analytical method for "fingerprinting" of organic compounds.

Over the past few decades, the application of this technique has opened up for new directions in analytical science and technology. Its use in combination with chemometrics led to new approaches in quality control and assurance, safety, and traceability as well as tackling challenges for analyzing innovative and established model systems in their totality.

This special issue aims at providing an overview of the latest developments in the field of mid- and near-infrared spectroscopy using ATR and transmission modes. A particular focus lies on applications in the design and development of new drugs, dietary supplements, botanicals, and nutraceuticals as well as in process development for production and quality assurance and in potential uses for labelling.

All articles, which are part of this special issue, reflect modern trends and outline new ideas for future applications of spectroscopic technologies combined with chemometric data analysis in the food and pharmaceutical sectors.

The special issue contains three review articles and three regular papers. The paper of $\mathrm{M}$. Zhu et al. reviews the quality control of tea by near-infrared (NIR) reflectance spectroscopy and chemometrics. Tea is one of the most popular beverages around the world. The wide variety of tea quality, however, calls for measures of control quality for commercialized tea products. NIR reflectance spectroscopy is a rapid, noninvasive, and nondestructive tool that required minimal sample preparation. The article gives an overview of recent advances and applications in this field. The review by C. HernándezAguilar et al. addresses the application of the photoacustic spectroscopy (PAS) to evaluate of the quality of food. The PAS technique is based upon photothermal phenomena, which allow spectroscopic studies. An overview is given on PAS applications in the food industry, for example, to analyse fruit, vegetables, condiments, grains, milk, water, eggs, and so on. It was concluded that the method is capable of detecting adulteration and contamination of food samples. The third review article by S.A. Mahesar et al. is concerned with the applications of NIR and MIR spectroscopic techniques to olive oils. A special focus of the paper is the detection and determination of functional compounds such as fatty acids and phenols. It is demonstrated that MIR spectroscopy is particularly well suited to distinguish olive oils from potential adulterants. The contributed regular papers demonstrate cutting-edge advancements of the field. G. Shi et al. described real-time release testing of herbal extract powder by nearinfrared spectroscopy considering the uncertainty around specification limits. For two active pharmaceutical ingredients (APIs), they show that the reliable concentration ranges covering the specification limits can be verified by the accuracy 
profile (AP) methodology. The method was tested in the estimation of the $\beta$-content and $\gamma$-confidence tolerance intervals ( $\beta$-CTIs) around the specification limits. H. Ouhaddouch et al. applied FT-IR spectroscopy for the identification of a mineral drug substance in drug products using a case study on bentonite. For this purpose, they carefully characterized purified bentonite, bentonite in organic mixtures and organic excipients, and mineralized organic mixtures containing bentonite. They observed distinct changes and variations in the spectra, which could be attributed to the properties of the samples. $\mathrm{H}$. Fu et al. reported the use of an NIR-MIR data fusion technique and evaluated the spectra via a moving window partial least-squares discriminant analysis for authenticity and adulteration discrimination of herbs. They concluded that their approach is a promising tool for the rapid discrimination of geoherbalism and authenticity of Chinese herbs. We hope that the readers will find this special issue interesting and inspiring.

\section{Conflicts of Interest}

The editors declare no conflicts of interest.

\section{Acknowledgments}

We would like to thank the authors and the reviewers of the publications in this special issue for their invaluable contribution and effort. We are also grateful to the editorial board members and support staff of the journal for their kind support during the preparation of this special issue.

\section{Alessandra Durazzo \\ Massimo Lucarini Johannes Kiefer}

Sarfaraz Ahmed Mahesar 